of the vertebræ. In a later article* written mainly in review of Repossi's paper on Mixosaurus, Yakowlew expresses the opinion that the double-headed character appeared first in the posterior portion of the dorsal region owing to the more vigorous movement of that portion of the body. It is difficult to reconcile this theory with the fact that in Ichthyosaurus the ribs tend to be single-headed toward the base of the tail and double-headed anterior to it, and that in Shastasaurus, which had also a great sculling tail, the ribs are all singleheaded excepting a very few immediately behing the head.

Concerning Yakowlew's suggestion that the single-headed rib is the primitive type in this order, it is certainly a significant fact that it seems to occur frequently in the Triassic ichthyosaurs. We should not forget, however, that the oldest representative of the order which has been described, viz., Quenstedt's atavus, from the lower Muschelkalk, is said to have a double articulation and is possibly not to be referred to Mixosaurus, as has been generally supposed. Recent discoveries have shown the existence also in the Californian Triassic fauna of a form (Toretocnemus $\dagger$ ), in which the middle dorsal ribs are as widely forked as in Ichthyosaurus. Again, it appears that in Shastasaurus the double articulation in the anterior part of the column is not formed by reduction of the middle portions of simple lateral apophyses. From the atlas to the anterior dorsal region the diapophyses are gradually increased in height and the parapophyses reduced till the latter are mere points some distance below the lower ends of the diapophyses. The lateral apophyses of the dorsal region, therefore, correspond to the diapophyses in the cervical region. If the double articulation in the neck region is secondary it would appear to have required the addition of a lower rib head and a parapophysis. There appears, therefore, to be still a chance that the double articulation is

* Einige Bemerkungen ueber die triassischen Ichthyosaurier.' Verhand. der Kais. Russ. Mineralog. Ges., Bd. XL., Lief II.

† See Bull. Geol. Dept. Univ. of Calif., Vol. 3, No. 12, p. 260. primitive in this group, and it may be well to withhold final judgment till we have a better acquaintance with the structure of the vertebral column as a whole in the later Triassic forms, and particularly till we know more about the Middle Triassic representatives of the group.

John C. Merriam.

\section{NOTES ON PHYSICS.}

INTERFERENCE OF LIGHT WITH GREAT PATH DIFFERENCE.

The most carefully designed and constructed mechanical vibrator, such as a pendulum, cannot be made to vibrate, when left to itself, more than a few thousands of times without greatly decreasing in amplitude. On the other hand, the number of free oscillations made by an atom of a luminous gas during the intervals between collisions between that atom and others, during which times the atom is presumably not receiving energy from any source to make good its losses by radiation, is, in the case of mercury vapor at least, as many as 2,600,000 and that without very great decrease of amplitude. This follows from some recent work of Lummer and Gehrcke, who have recently shown that a given portion of the beam of light from mercury vapor (the green rays) is in condition to interfere with a portion of the same beam 125 centimeters or $2,600,000$ wavelengths farther along the beam, which shows that as many as 2,600,000 successive waves come without a break of continuity from the vibrating luminous particles in mercury vapor.

\section{THE ELECTROMAGNETIC THEORY OF MATTER.}

The most complete and readable presentation hitherto made of the mathematical theory of the motion of minute electric charges (electrons) is given by Max Abraham in Drude's Annalen for January, 1903. It is by comparison of the results of this mathematical theory with results of experiments on cathode and Becquerel rays, on the Zeeman effects, etc., that the electromagnetic theory of matter has arisen.

An electrically charged body in motion has more momentum and stores more energy, in 
other words it has apparently a greater inertia mass, than the same body without charge, and the electrical part of the mass is greater and greater the smaller the size of the body. The idea, which is fast gaining strength, that atoms are aggregates of extremely small charged particles, corpuscles as J. J. Thomson calls them, has given credit to the hypothesis that inertia is wholly electrical in character, inasmuch as an atom is known not to be small enough, assuming it to be homogeneous, to permit of its inertia being attributed wholly to its electric charge. The word electron seems to be most acceptable as a name for these ultimate corpuscles of disembodied electric charge.

The 'mass' of an electron is not fixed in value but it increases with velocity. In fact the very rapidly moving electrons (95 per cent. of the velocity of light) in Becquerel rays have greater mass than the more slowly moving ions in cathode rays, and measurements of mass and velocity of the electrons in cathrode rays and in Becquerel rays are in accord with the theoretical law of increase of mass with velocity. When an electron is accelerated in the direction of its motion its mass is different (longitudinal mass) from when it is accelerated at right angles to its direction of motion (transversal mass). The difference between longitudinal and transversal mass is sufficiently great numerically, when the velocity is great, to be an essential element in the established correspondence between theory and observation above mentioned.

The inertia or mass of the electron is purely electromagnetic in character; and if atoms and molecules are mere aggregates of electrons it follows that all mass and inertia are electromagnetic in character.

Some very curious laws of motion arise from the electron theory. When velocities and accelerations are very small Newton's laws of motion apply as very close approximations. When velocịties or accelerations are large Newton's laws of motion fail utterly. One point of failure is the above-mentioned distinction between longitudinal and transverse mass-mass is not a scalar quantity but a so-called linear vector function; other curious points in the motion of electrons are the following:

Uniform motion in a straight line once established is steady or permanent. Uniform motion of an electron in a circular orbit, on the, other hand, is accompanied by continuous radiation of energy, so that such motion must eventually cease if it is not maintained by some external agent. Thus two electrons rotating about each other after the manner of planet and satellite slowly radiate energy, and their motion is affected very much as if they were in a resisting medium. This motion of electrons as satellites is the fundamental hypothesis in the theory of the Zeeman effect.

A continuously accelerated electron stores an increasing amount of kinetic energy which can be regained by stopping the electron, and in addition it radiates energy continuously, which energy is lost. If the acceleration is very great this radiation may be considerable. Therefore, there is a drag upon the electron which is something like viscous friction but which depends upon acceleration and not on velocity.

An electron under way will, if quickly stopped and released, start up again of itself and move with diminished velocity in the original direction.

When an electron gains a certain velocity under the action of an accelerating force and the force suddenly ceases to act, then the electron loses some of its velocity and settles down to a slightly decreased uniform velocity.

An electron started quickly and kept moving at uniform speed must be acted upon, of course, by a great force to produce the quick start, and also by a lasting force which slowly drops to zero.

The kinetic energy of an electron is not proportional to the square of its velocity. This is another way of stating the fact which lies at the foundation of the variation of mass with velocity, and of the distinction between longitudinal and transverse mass. Thus, the only case in which an added velocity $Y$ in the direction of the $y$-axis of reference means the same amount of work done whether 
the moving particle has or has not already a velocity $X$ in the direction of the $x$-axis, is the case in which the kinetic energy is simply dependent upon $X^{2}+Y^{2}$.

Not directly connected with this matter of the dynamics of the electron, but of great interest, is the question of the amount of electrical energy stored in the electric field which surrounds an electron. Taking the data which have been obtained from experiments on cathode rays, it appears that a number of electrons sufficient to weigh a gram have associated with them about $6.10^{13}$ joules (or 40 million million foot-pounds) of energy when stationary. That is, estimating that a hot gas radiates 5 watts per gram, the total electron energy associated with an atom would last for a hundred million million million oscillations of full intensity before it were all exhausted by radiation, or rather the energy associated with a pair of rotating electrons would last for a hundred million million million periods before it were all radiated. This gives us some insight into the matter mentioned in the foregoing note on the interference of light with great path difference. It is probable that the limit of path difference which will produce interference is determined by frequency of molecular collisions rather than by diminished amplitude of atomic oscillations between collisions.

Another matter of interest, growing out of the excessively small size of the electron, vastly smaller than the atom, is that the electrons are always at great distances apart in comparison with their size, so that the variations of total energy due to different forms of electron aggregation are but a small fraction of the total energy. Thus the diminution of energy accompanying the formation of a gram of water is only about 16,000 joules or one four-thousand-millionth part of the total electron energy.

Another matter of interest is that the volume integral of ether stress-which may be the fundamental cause of gravitation-is independent of states of electron aggregation to about the same degree of approximation as above pointed out for electron energy.

W. S. F.

\section{CURRENT NOTES ON METEOROLOGY.}

\section{MARCH WEATHER PROVERBS.}

Mr. B. C. Webber, acting director of the Meteorological Service of Canada, contributes a paper on 'March Winds' to a recent number of the Monthly Weather Review (Vol. XXXI., No. 3). On the basis of thirty years' records for Ontario, Quebec and the Maritime Provinces, it appears that there is a considerable decrease in the number of days with high winds in March, as compared with the three preceding months; that the month of March has fewer cold winds than February; that the snowfall of March is very much less than during the preceding winter months, and that there is nothing in the records for the past thirty years to justify the assumption that 'if March comes in like a lion it goes out like a lamb.' Thus do many of the 'popular' sayings about the weather often prove to be without foundation in fact when they are confronted with the results of meteorological observations. The results of the investigation along somewhat similar lines, by W. B. Stockman, of the U. S. Weather Bureau, are discussed in the May Review.

\section{HEIGHT OF THE SEA BREEZE.}

Observations as to the height of the diurnal sea breeze are few in number, albeit of considerable importance. By means of a captive balloon, sent up from Coney Island a number of years ago, it was found that the average height at which the cool inflow from the ocean was replaced by the upper warm outflow from the land was from 500 to 600 feet. At Toulon, in 1893, the height of the sea breeze was found to be about 1,300 feet, and a distinct off-shore current was found between 1,900 and 2,000 feet. More recently (1902), on the west coast of Scotland, Dines, using kites, has noted that the kites would not rise above 1,500 feet on sunny afternoons, when the on-shore breeze was blowing (Quart. Journ. Roy. Met. Soc., April, 1903).

STORMS OF THE GREAT LAKES.

Fon some years the Weather Bureau has been giving special attention to the storms which occur over the Great Lakes, with a view 